\title{
Evolution Corundum Based Detector in UV and VUV Spectral Region
}

\author{
Vardanyan $\mathrm{J}^{1 *}$, Hovhannisyan $\mathbf{M}^{1}$, Agajanyan $\mathbf{H}^{1}$ and Nersisyan $\mathbf{M}^{2}$ \\ ${ }^{1}$ Institute of Applied Problems of Physics, RA Academy of Sciences, Armenia \\ ${ }^{2}$ Institute of Physical Research, Armenia
}

*Corresponding author: Vardanyan Julia, Institute of Applied Problems of Physics, RA

\section{Research Article \\ Volume 3 Issue 3}

Received Date: August 20, 2019

Published Date: August 30, 2019 Academy of Sciences, Erevan, Armenia, Tel: +37493975257; Email: julia.vardanyan2011@yandex.ru

\section{Abstract}

The UV and VUV spectral range of photons is an important area for experimental studies in the field of life sciences and materials science, especially for studies of large molecules, crystals, surface science, etc. Currently, all synchrotron radiation facilities have a special program of experimental studies in the field of VUV spectrum. Large-format ultraviolet image sensors have been and are being actively developed for various space astronomical missions. The detector plays a crucial role in the overall capabilities of the device. There are many detector systems, none of which are ideal for all applications. The wavelength ranges of interest are 10-200 nm for studies in solar physics, $280 \mathrm{~nm}$ for communications and missile warnings, and 200-400 nm for studies of the atmosphere. Compactness, high sensitivity, durability and reliability are critical requirements for space applications. One of the earliest methods used to record a system (detection, visualization) - a system based on a scintillator of ionizing radiation (image scanners) remains one of the most flexible and successful methods. However, conventional scintillators have significant limitations: degradation in vacuum, limited point spread function (PSF) (usually> 100 microns FWHM) due to scattering on the screen of a polycrystalline phosphor. Solid scintillation films are ineffective because typically $>90 \%$ of the light is captured on the screen by total internal reflection, i.e. $<10 \%$ of the light is emitted. The partial solution to improve the spatial resolution of the detecting system is the usage of rigidly structured scintillator, for example, fiber, needle. The fiber-optical systems are widely used in life and material sciences, bio and nanotechnology. We conducted numerous spectral studies with multiple corundum crystals (with various impurities and their concentrations, as well as their ratios $\mathrm{Cu}, \mathrm{Fe}, \mathrm{Ga}, \mathrm{Ge}, \mathrm{Mg}, \mathrm{Mn}, \mathrm{Ni}, \mathrm{Pb}, \mathrm{Se}, \mathrm{Si}, \mathrm{Ti}, \mathrm{V}, \mathrm{Zn} . \mathrm{Sc}$. In the course of work, the crystal samples were subjected to technological processing methods that allow changing the optical density of corundum in order to identify the mechanisms of energy transfer and the optimal use of crystals as sensors for VUV, UV, and IR radiation. As a result of the studies, those substances, their concentrations and ratios that have resonance absorption lines not only in the UV, but also in the VUV spectral regions were identified. Such resonant lines have lanthanum and lanthanides, scandium and others. Theoretically, this is understandable: having large atomic radii, they can create new exciton-photon interactions, which lead to new transitions and energy transformations. 


\section{Physical Science \& Biophysics Journal}

Keywords: Detector; Ultraviolet radiation; Impurities; Corundum; Luminescence; Optical fiber

\section{Introduction}

Large-format ultraviolet image sensors have been and are being actively developed for various space astronomical missions. The detector plays a crucial role in the overall capabilities of the device. There are many detector systems, none of which are ideal for all applications. The wavelength ranges of interest are 10$200 \mathrm{~nm}$ for studies in solar physics, $280 \mathrm{~nm}$ for communications and missile warnings, and 200-400 nm for studies of the atmosphere. Compactness, high sensitivity, durability and reliability are critical requirements for space applications [1-3].

Our goal: to create a detector based on corundum, based on scintillation, which in its characteristics can complement the existing drawback.

It is assumed that in the range of 50-400 nm there are two possible converter circuits - a single-crystal converter (ordinary) and a system converter with a "fiber bundle" similar to a fiber-optic front panel. An improvement in the luminescent photon registration system will make the detector also position-sensitive.

\section{Materials and Methods}

The study was carried out in some areas [4].

1. Experimental studies of the sensitivity of new and fiber crystals based on corundum with activated impurities,

2. Comparative study of the spectral sensitivity of crystals and determination of optimal crystal compositions with an extended spectral range,

3. Development of a binder system for crystalline fibers [5].

The measurements of the absorption ability of the crystals have been performed on setups UV/VIS and M400 spectrometers, the luminescence ability has been measured by Perkin-Elmer Model Mpf-44B.

Several corundum crystals with impurities of interest (3 pieces) were selected, the properties of which more or less correspond to our requirements (Figure 1).

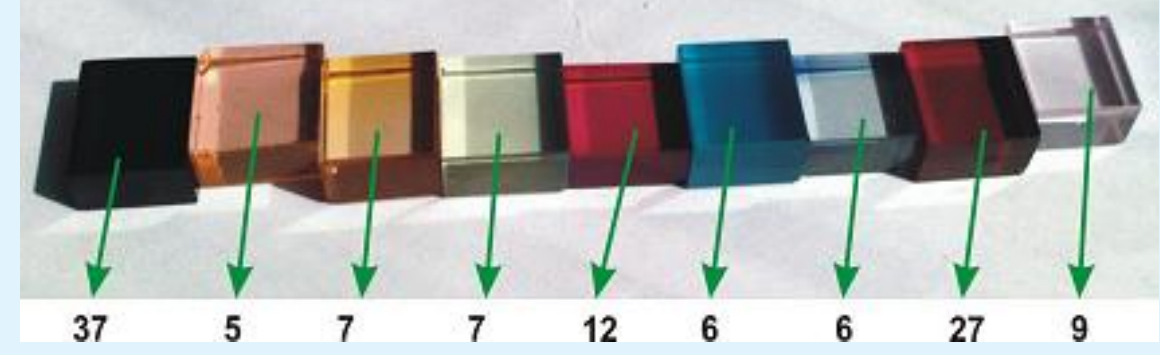

Figure 1: The numbers of crystals are the values of relative luminescence intensity in mV measured by a silicon solar cell.

On the AMPTEK X-ray monochromator, graphs of concentration and their ratios were obtained (Figures 24).

Very dark vinous: Al-97.88322, $\mathrm{Cr}-1,97 \%, \mathrm{Mn}-0,087 \%$, $\mathrm{Zn}-0,027 \%$, Ti-0,022\%, Fe-0,0057\%,Cu-0,003\% , $\mathrm{Cr}_{2} \mathrm{O}_{3}+$ $\mathrm{TiO}_{2}+\mathrm{Fe}_{2} \mathrm{O}_{3}$ ), $\mathrm{Sc}-0,002 \%$
Light pink: Al-99.92875, Cr-0.027139\%, Mn-0.000885\%, Zn- $0.002189 \%$, Ti-0.011242\%, Fe- $0.00658 \%$,Cu$0.012847 \%$

Orange-Yellow: Al-99.31313, Cr-3.938606E-02\%, Mn2.666649E-02\%, Zn-0.005442148\%, Fe-4.473137E-02, $\mathrm{Ni}-3.188814 \mathrm{E}-02$ 


\section{Physical Science \& Biophysics Journal}

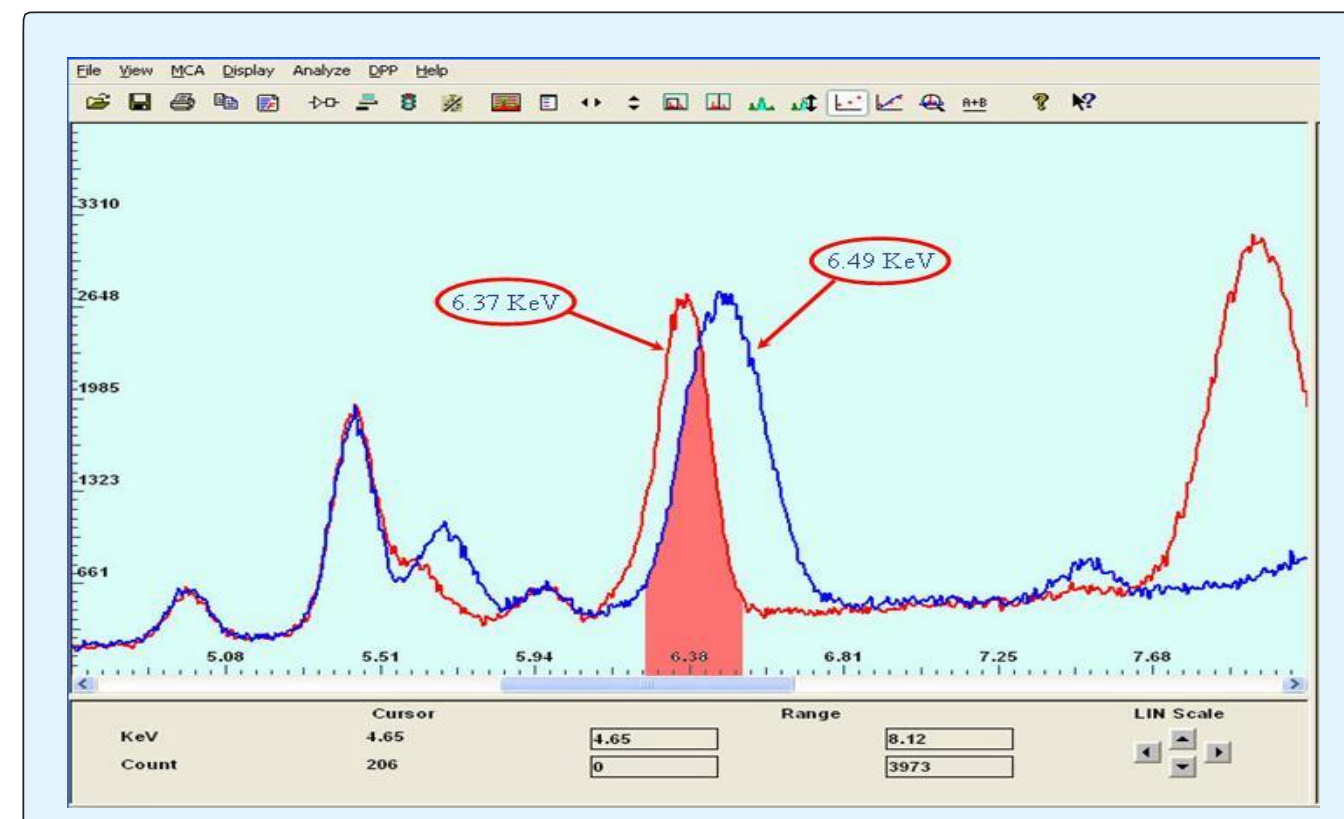

Figure 2: Displacement and extra peaks.

It was shown that additional peaks appear in the luminescence spectra of such samples, the energies of which sometimes differ significantly from the energies of the peaks of chemical elements. In some cases, these peaks partially or fully cover the peaks of the elements. All this leads to significant errors in determining the composition of crystals.
Additional studies were carried out - when the sample rotated around its axis, and possible factors affecting the measurements were identified and corrected. The main conclusion is that, in the study of single crystals, in addition to luminescent peaks, diffraction peaks can also be detected - the so-called "parasitic" peaks.

\begin{tabular}{|c|c|c|c|c|c|c|c|c|c|}
\hline & \multicolumn{9}{|c|}{ Sample } \\
\hline Element & No1 & No4 & No5 & No7 & No8 & $\begin{array}{c}\text { corundum } \\
(25 \mathrm{KeV})\end{array}$ & $\begin{array}{c}\text { Ruby } \\
\text { natural }\end{array}$ & $\begin{array}{c}\text { Ruby natural } \\
\text { (with K) }\end{array}$ & $\begin{array}{c}\text { corundum } \\
\text { white }\end{array}$ \\
\hline "Al" & 99.6146 & 99.4299 & 98.8347 & 98.6095 & 98.8347 & 99.7539 & 96.2494 & 94.46476 & 99.7417 \\
\hline "Sc" & - & $1.09 \mathrm{E}-02$ & - & - & - & - & - & - & - \\
\hline "Ti" & $9.03 \mathrm{E}-03$ & - & - & $1.60 \mathrm{E}-02$ & - & - & 0.35254 & 0.44435 & $7.19 \mathrm{E}-04$ \\
\hline "V" & $1.57 \mathrm{E}-03$ & 0.06795 & 0.31668 & $8.92 \mathrm{E}-02$ & 0.31668 & - & 0.17215 & 0.26881 & - \\
\hline "Cr" & $8.05 \mathrm{E}-02$ & 0.25776 & 0.46574 & 0.48724 & 0.46574 & $3.64 \mathrm{E}-02$ & 1.34435 & 1.83645 & $3.37 \mathrm{E}-02$ \\
\hline "Fe" & $3.38 \mathrm{E}-02$ & $6.43 \mathrm{E}-02$ & $7.66 \mathrm{E}-02$ & $3.55 \mathrm{E}-02$ & $7.66 \mathrm{E}-02$ & $1.58 \mathrm{E}-02$ & 1.52497 & 2.35893 & $1.83 \mathrm{E}-02$ \\
\hline "Co" & $2.69 \mathrm{E}-02$ & $1.65 \mathrm{E}-02$ & 0.10648 & 0.15392 & 0.10648 & - & - & - & $2.52 \mathrm{E}-02$ \\
\hline "Ni" & - & - & - & $8.89 \mathrm{E}-03$ & - & $7.05 \mathrm{E}-03$ & 0.13923 & 0.23894 & - \\
\hline "Cu" & - & - & - & - & - & - & $8.24 \mathrm{E}-02$ & 0.14544 & 0.05507 \\
\hline "Zn" & 0.01469 & $1.42 \mathrm{E}-02$ & $3.86 \mathrm{E}-02$ & $1.58 \mathrm{E}-02$ & $3.86 \mathrm{E}-02$ & 0.10112 & $4.92 \mathrm{E}-02$ & $8.50 \mathrm{E}-02$ & - \\
\hline "Ga" & $1.49 \mathrm{E}-02$ & - & $9.24 \mathrm{E}-02$ & $7.11 \mathrm{E}-02$ & $9.24 \mathrm{E}-02$ & - & 0.04036 & $7.57 \mathrm{E}-02$ & - \\
\hline "As" & 0.15426 & 0.11706 & - & 0.51287 & - & $5.09 \mathrm{E}-02$ & - & - & 0.01223 \\
\hline "Sr" & - & - & - & - & - & $9.61 \mathrm{E}-03$ & - & - & - \\
\hline "Se" & $4.97 \mathrm{E}-02$ & $2.13 \mathrm{E}-02$ & $6.89 \mathrm{E}-02$ & - & $6.89 \mathrm{E}-02$ & - & $4.54 \mathrm{E}-02$ & $8.15 \mathrm{E}-02$ & - \\
\hline "Mo" & - & - & - & - & - & $1.75 \mathrm{E}-02$ & - & - & - \\
\hline
\end{tabular}

Table 1: Dopant samples composition and concentration: measurements by AMPTEK equipment. 


\section{Physical Science \& Biophysics Journal}

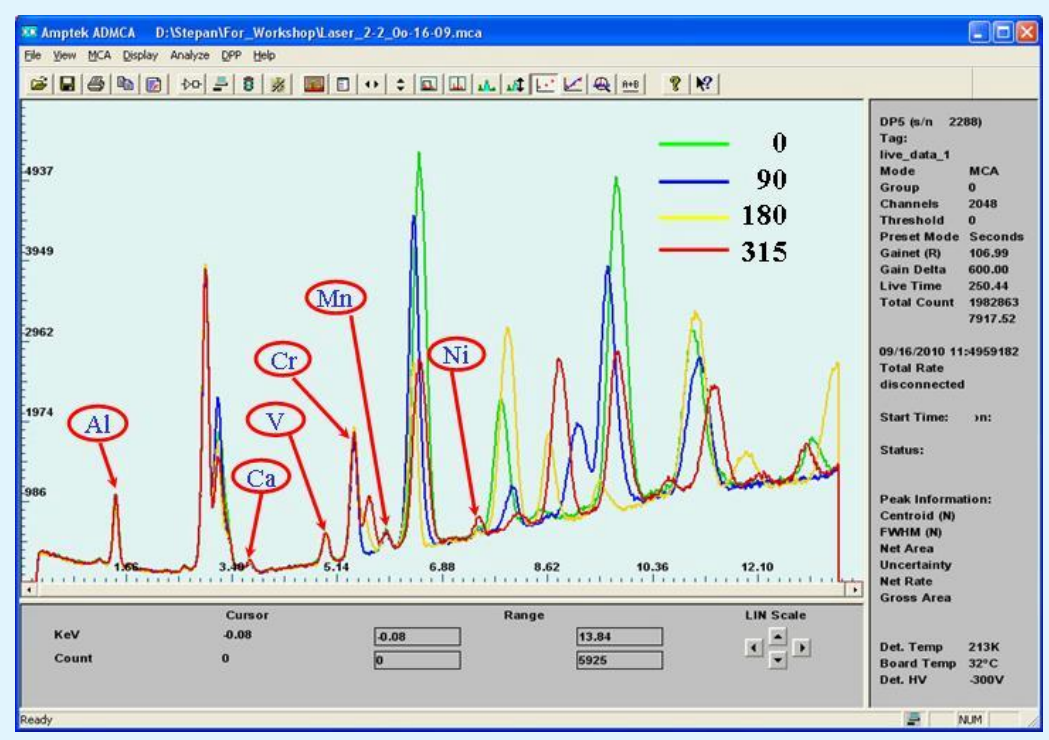

Figure 3: Fluorescence spectra in different orientations of the sample.

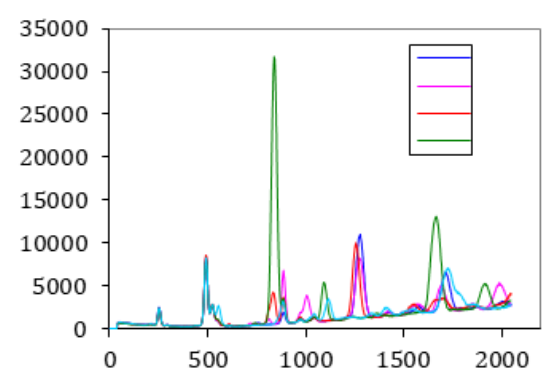

Figure 4: Mapped spectral graphs of the samples in Table 1.

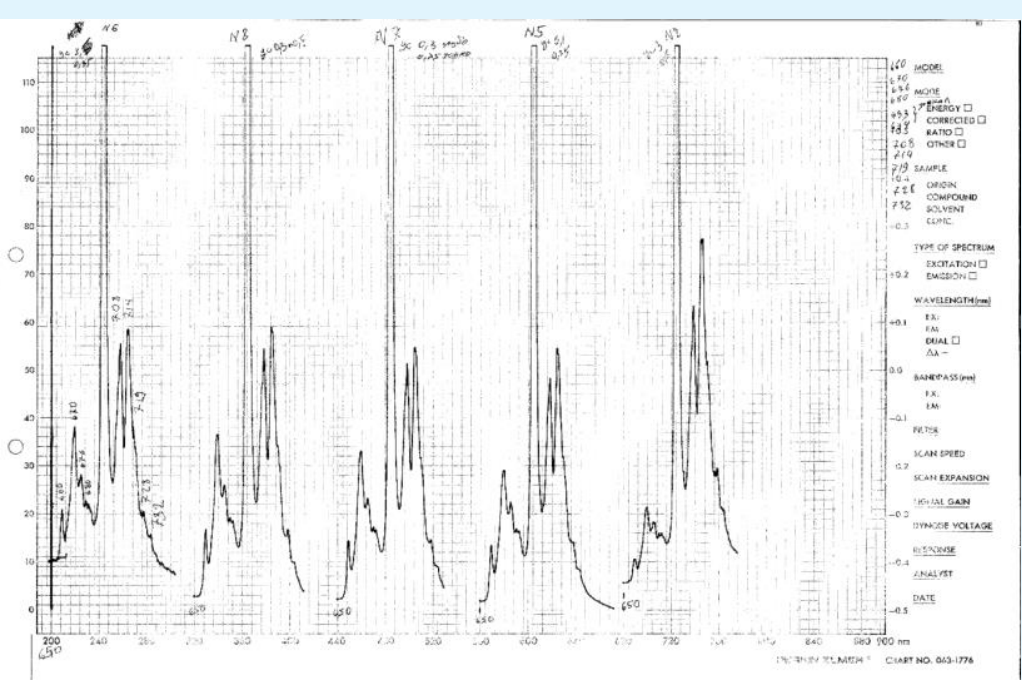

Figure 5: Luminescent spectra curves of crystals of Table 1. 
The analysis of experimental data has shown that the crystal very dark vinous has the most intense luminescence. As it can be seen from the spectrum in
Figure 6 the crystal fluoresces only in the wavelength range from $660 \mathrm{~nm}$ to $790 \mathrm{~nm}$, and those lines are caused not only by chrome.

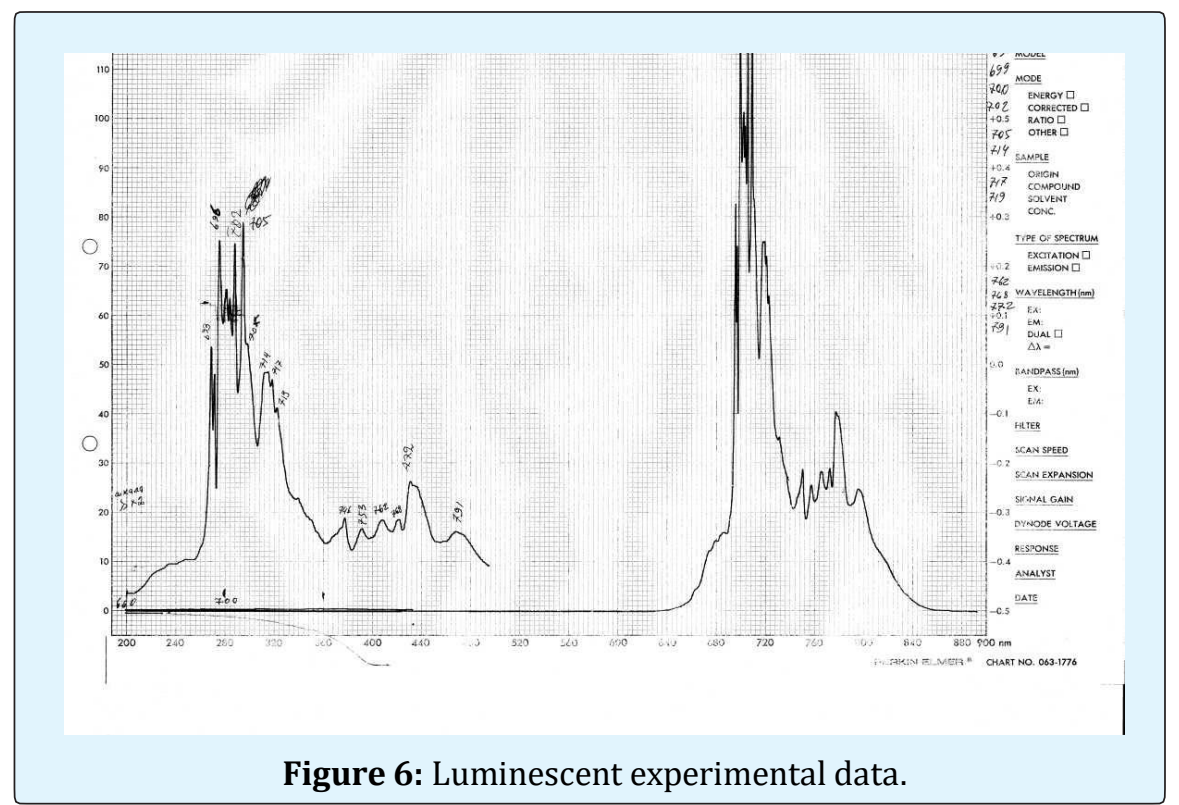

The luminescence of a relatively pure ruby with caused only by chrome is presented in Figure 7. characteristic bands 240-250, 300-540 $\mathrm{nm}$ and R-lines

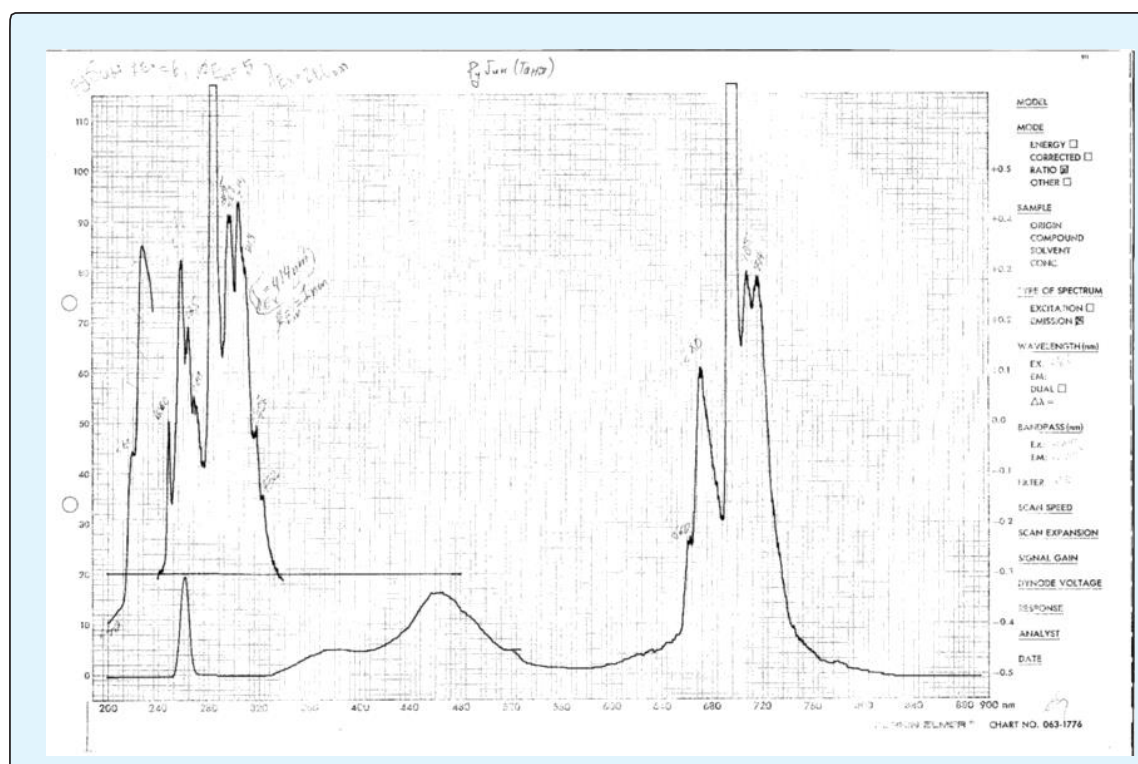

Figure 7: The luminescence spectrum ranges.

The luminescence intensity of the selected very dark burgundy corundum crystal (Figure 6) is 30\% higher than the intensity of pure ruby (Figure 7) (the luminescence spectrum is concentrated only in the range of 660-790 $\mathrm{nm}$, respectively, and the integrated intensity is measured) 


\section{Physical Science \& Biophysics Journal}

The luminescence spectra at different wavelength of excitation radiation for very dark vinous crystal have also been investigated (Figure 8-11).

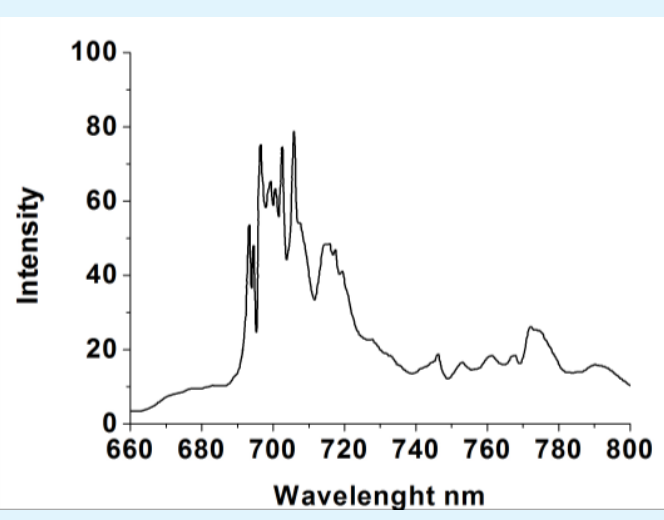

Figure 8: The luminescence emission spectrum at excitation with $468 \mathrm{~nm}$ wavelength.

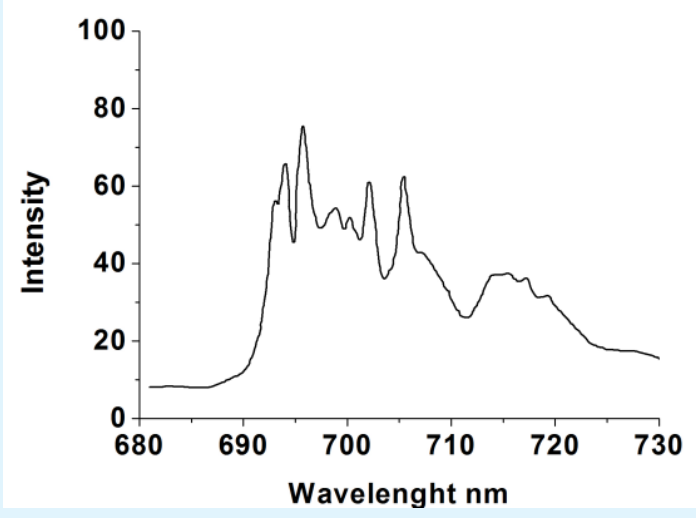

Figure 9: The luminescence emission spectrum at excitation with $370 \mathrm{~nm}$ wavelength.

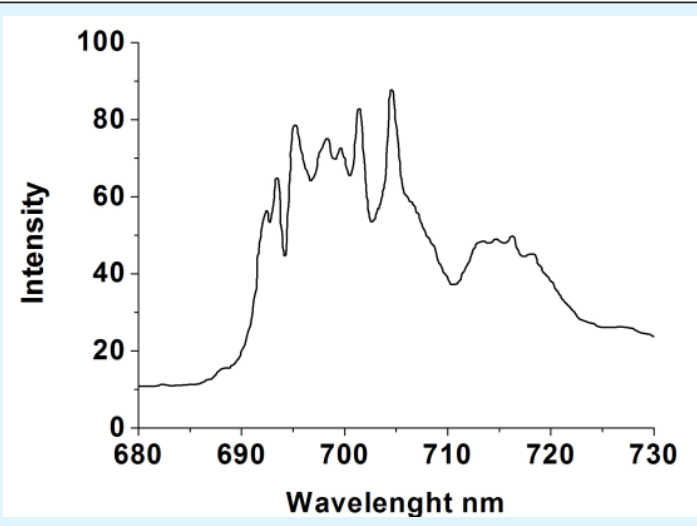

Figure 10: The luminescence emission spectrum at excitation with $357 \mathrm{~nm}$ wavelength.

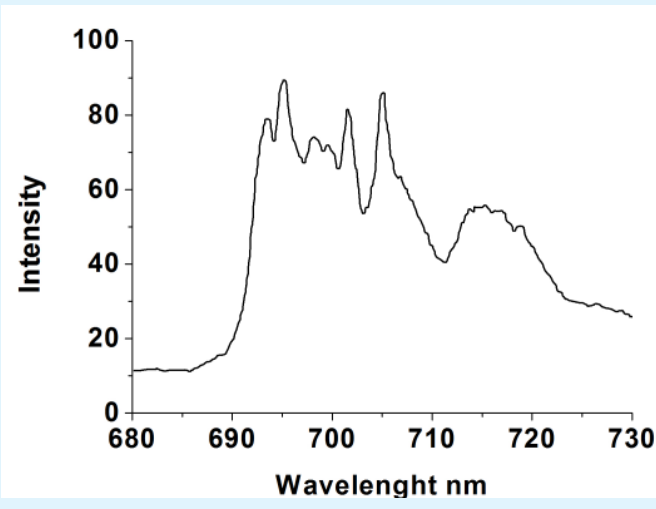

Figure 11: The luminescence emission spectrum at excitation with $300 \mathrm{~nm}$ wavelength.

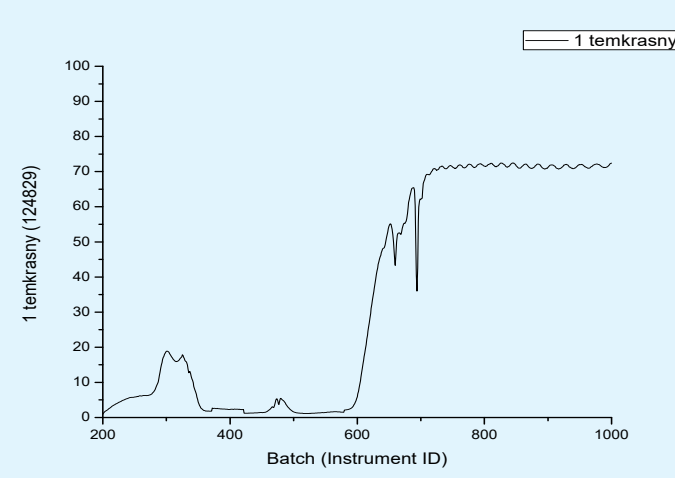

Figure 12: Very dark vinous- transmission.

Corundum samples with the same dopant concentrations and absorption properties were annealed (in a limited amount). Annealing temperature of 3001000C was chosen.

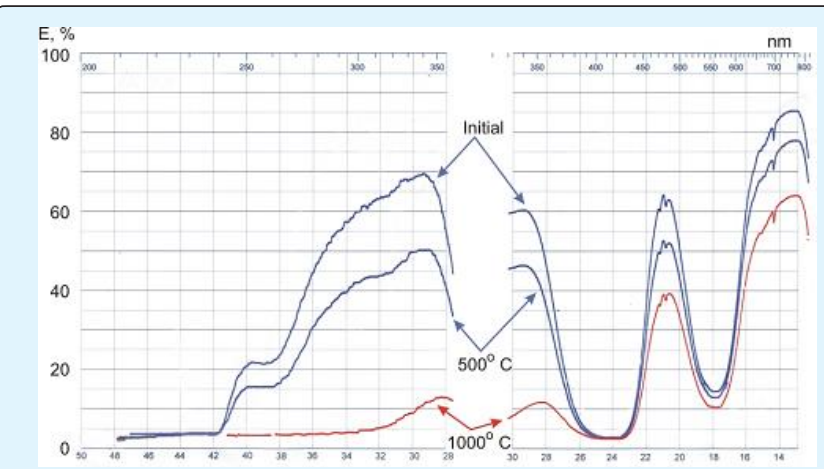

Figure 13: UV-VIS spectrometer measurement graphs. 


\section{Physical Science \& Biophysics Journal}

A graph of the absorption properties of the sample measured by the UV-VIS spectrometer before and after annealing is shown in Figure 13. Nevertheless, corundum samples with the same dopant concentrations and absorption properties were selected, then some of them were rejected due to visible defects (measurements under a microscope) and the process of annealing was started. Annealing temperatures of 300500 and $1000 \mathrm{C}$ were selected.

This typical picture of the transmission spectra before and after thermal annealing is even much complicated, since the behavior of the sample resembles a superposition of many fairly close maxima-the absorption band, both by structural defects and by impurity ions, which are strongly dependent on temperature.

It can be seen that with increasing annealing temperature, the throughput begins to decrease not monotonously, impurity defects are more stable, recharging or energy transfer to the crystal lattice can take place, but absorption bands as a whole decrease in the bulk of samples with different speeds and kinetics. In any case, upon annealing, the optical properties of crystals change. The annealing method was used to change the optical properties of crystals. The spectral characteristics of the sapphire short-wavelength absorption edge lie in the vacuum ultraviolet region between 142 and $145 \mathrm{~nm}$, the entire transparency region is well studied, and it is believed that the absorption bands with maxima at $175,206,230,400 \mathrm{~nm}$ are due to structural centers of color (CES), impurity impurity color centers (CCR). Thermal annealing, radiation exposure (the main methods for generating defects, in particular, color centers) can be expanded to improve certain properties of crystals, so it was decided to try to change the properties of the samples under study by thermal annealing.

Two versions of a detection system in the UV and VUV range of the spectrum were developed, that is, two possible transducer designs - a single-crystal transducer (conventional) and a transducer system with a "fiber optic beam" similar to fiber optic front panel. To create a detector with spatial resolution (which is determined by the size of the element assembled into a mosaic), a crystal beam technology was developed. The operation sequence is similar to FOR (fiber optic plate) or MCR (multi-channel plate) technology $[6,7]$.

The improvement of the luminescent photons recording system will make the detector also positionsensitive.

\section{Results}

Eight crystals were selected for comparative measurements of the integrated luminescence intensity, and detectors were fabricated. Comparative measurements of the integrated luminescence intensity for eight crystals were carried out at the X-ray optics laboratory at the PPF Institute, and some studies were performed at DEZZY (Germany).

A laser with a pulse duration of $\sim 100 \mathrm{fs}$ and a frequency of $100 \mathrm{kHz}$ at two wavelengths of $400 \mathrm{~nm}$ and $266 \mathrm{~nm}$ was used as a radiation source. A pulse-fed xenon lamp (frequency $1 \mathrm{~Hz}$ ) after passing through a UFS2 filter is used as a source of ultraviolet radiation. The results obtained (integrated luminosity) correlate well with each other. As for measuring the duration of constancy, they differ in order, which may be due to the difference in the power and duration of the exciting radiation in the laboratories [8-10].

\section{Conclusion}

Single crystal samples with various dopants, their optical properties, and absorption and luminescence spectra were studied. Measurements of the luminescence spectrum of corundum are performed at several wavelengths within the capabilities of the available equipment. The limitations of the studies were caused, in particular, by the inability to obtain or acquire similarly oriented models of the samples in order to exclude the effects of polarization in the presence of selectively controlled additives of only one or two elements.

However, the crystals for the manufacture of the transducers were selected, and comparative measurements of the integrated luminescence intensity for the three crystals were performed in the X-ray optics laboratory at the Institute of Optical Physics. As for the image detector, $0.25 \times 0.25 \times 2.5 \mathrm{~mm}$ fiber crystals were prepared, the matrix was combined, and the images were obtained using webcams.

\section{References}

1. Reliability workshop, 4-6/2/2002, ESRF, Grenoble.

2. Graafsma $H$ (2006) Introduction to the Special Issue on Detectors. J Synchrotron Rad 13(2): 97-98. 


\section{Physical Science \& Biophysics Journal}

3. Monroy E, Palacios T, Omne`s F, Calle F, Hochedez JF (2002) Assessment of GaN metal-semiconductormetal photodiodes for high-energy ultraviolet photodetection. Applied Physics Letters 80(17).

4. Durst RD (2003) Advances in X-Ray Scintillator Technology. 13 the ESRF Users Meeting, Grenoble, France.

5. Rohr P (2003) Amorphous Silicon based matrix detectors for X-ray Imaging, 13 the ESRF Users Meeting, Grenoble, France.

6. Lopez-Higuera JM (2002) Handbook of Optical Fiber Sensing Technology. John Wiley \& Sons Inc., pp: 828.
7. Anbo Wang Optical Fiber Sensors and Systems. Center for Photonics Technology, Virginia Polytechnic Institute and State University Blacksburg, U.S.A.

8. Vardanyan J, Agajanyan H, Nersisyan M (2018) Receivers of VUV and UV Radiation. Armenian Journal of Physics 11(3): 141-144.

9. Vardanyan J, Agajanyan H, Nersisyan M (2019) Corundum Detector Converter. Armenian Journal of Physics 12(2): 213-217.

10. Aslyan Z, Madatyan K, Vardanyan J, Tsakanov V, Mikaelyan R (2006) Ruby based detector in the and VUV spectral region. Brilliant Light in life and Material Sciences, Yerevan, pp: 491- 494. 